Revista Destaques Acadêmicos, Lajeado, v. 11, n. 4, 2019. ISSN 2176-3070

DOI: http://dx.doi.org/10.22410/issn.2176-3070.v11i4a2019.2403

http://www.univates.br/revistas

\title{
ANALYSIS OF THE APPLICATION OF REINFORCEMENT LEARNING ALGORITHMS ON THE STARCRAFT II VIDEO GAME
}

\author{
Leandro Vian ${ }^{1}$, Marcelo de Gomensoro Malheiros ${ }^{2}$
}

\begin{abstract}
In recent years Machine Learning techniques have become the driving force behind the worldwide emergence of Artificial Intelligence, producing cost-effective and precise tools for pattern recognition and data analysis. A particular approach for the training of neural networks, Reinforcement Learning (RL), achieved prominence creating almost unbeatable artificial opponents in board games like Chess or Go, and also on video games. This paper gives an overview of Reinforcement Learning and tests this approach against a very popular real-time strategy game, Starcraft II. Our goal is to examine the tools and algorithms readily available for RL, also addressing different scenarios where a neural network can be linked to Starcraft II to learn by itself. This work describes both the technical issues involved and the preliminary results obtained by the application of two specific training strategies, A2C and DQN.
\end{abstract}

Keywords: Artificial Intelligence. Machine Learning. Neural Networks. Reinforcement Learning. Video Games.

\section{INTRODUCTION}

Artificial Intelligence (AI) has become part of our life, being a frequent term when computers are involved in automated processes of decision making, advancing in areas that were previously done only by humans. The recent resurgence of Artificial Intelligence, and particularly of its Machine Learning (ML) subfield, occurred when researchers combined techniques developed decades ago with modern parallel computers and programming techniques.

1 Graduate in Software Engineering at the University of the Taquari Valley - UNIVATES. leandrovian@hotmail.com

2 Professor at the University of the Taquari Valley - UNIVATES. PhD in Computing at UFRGS - RS, MSc in Electrical Engineering at UNICAMP - SP, graduate in Computer Engineering at UNICAMP - SP. mgm@univates.br 
Games are as old as humankind and have evolved hand in hand with civilization. With the advent of computers, they became complex pieces of software, allowing for a multitude of genres to be developed, such as roleplaying, first-person shooters and strategy video games.

As many computer games offer a simulated and self-contained environment, they are very useful as targets for Artificial Intelligence research, having a variety of challenges that need to be understood and overcome to achieve victory. Those test chambers can help train and validate automated learning and decision-making strategies that would otherwise be too complex or expensive to be solvable directly in a real-world environment.

In this work, we describe the application of current techniques of Machine Learning into the construction and validation of self-learning neural networks, by employing Reinforcement Learning algorithms.

We are particularly interested in a new environment that was created to help close the gap between video games and research: the SC2LE (Starcraft II Learning Environment), created in partnership by DeepMind and Blizzard Entertainment. This environment makes possible the development of an AI agent that plays the Starcraft II game. Such agent will then be capable of: controlling hundreds of units in cohesion, using strategy to exert influence and secure areas on the map; managing resources required to construct a base and build units; and making decisions based on imprecise information offered by the game screen and mini-map, both which have a "fog-of-war" effect that prevents vision if no friendly unit is nearby an area. Moreover, maps are big and diverse, offering many obstructions, besides the concept of land and air units. Finally, games may last for many minutes and decisions may not show results or consequences until later on.

All points considered, SC2 games are a difficult challenge towards building better AI tools and techniques that can be later applied to many areas, raising the simulation aspect of games to a new standard and importance.

The benefits that can be achieved by merging the amount of data available with discoveries in Reinforcement Learning are vast, where most areas can benefit in some manner by either having tasks being automated or simply by making better decisions in their processes. One example is in the medical area, where images, laboratory results and symptoms can go through AI systems that have been trained with millions of cases, helping doctors make decisions. Or maybe in simpler cases, where trained AI systems can help companies run more efficiently, lowering costs and improving results.

In the following sections, we describe our particular experiments, where we compare the A2C and DQN Reinforcement Learning algorithms, by creating agents that play two distinct Starcraft II mini-games. Different configurations and parameters are then analyzed, giving the basis for our results and conclusions. 


\section{LITERATURE REVIEW}

This section presents the main concepts used during the development of this work.

\subsection{Artificial Intelligence}

In the last two decades, AI has evolved a tremendous amount, stopping from being something that would be talked in academic circles or science fiction movies, to becoming part of our daily routine. Things such as personal voice assistants, self-driving vehicles or recommendation systems started to permeate all aspects of contemporary life (ADAMS, 2017).

Some interesting advancements can be pointed out, such as the one that resulted in the creation of a new business model that competes with the traditional taxi companies. The Uber company offers their clients the ability to ask for a ride knowing exactly how much they will have to pay, and how long will take for the car to arrive and for the trip to complete. This is done with the use of an AI system that incorporates specific behaviors that calculate distances, car availability, traffic and ride demands during specific times of the day. Another example of the regular use of AI in our routines be can be seen in the airline companies, which uses AI autopilots as a common tool and as much as possible, to many passengers surprise as noted by (NARULA, 2017).

Despite the diversity of technical advancements making headlines on news around the Internet, most of the time they are referred just as AI for the sake of simplicity, helping readers associate the information with the overall area of expertise. AI is a huge area of study which encompasses many fields (ATTICK, 2016). A representation of AI and its major fields can be seen in Figure 1.

Figure 1: Breakdown of AI and its major fields.

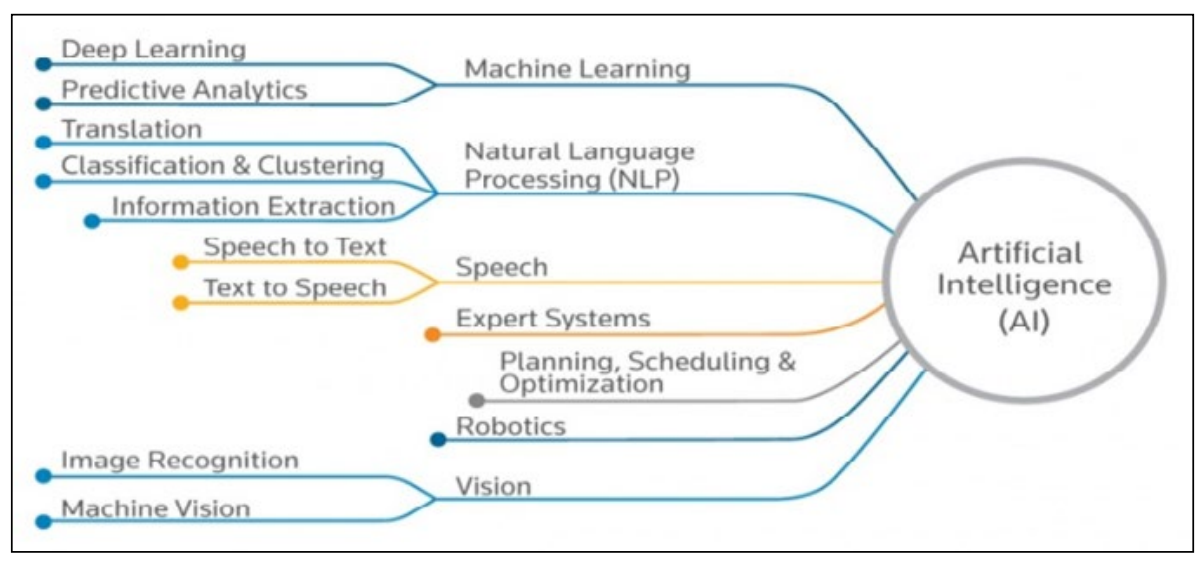

Source: (ATTICK, 2016) 


\subsection{Machine Learning}

Machine Learning is a field that is attracting a lot of current interest, being responsible for many recent advancements. This term was coined by Arthur Samuel in 1959, known for the creation of the world's first self-learning program. The program he developed played checkers and used a search tree of the board to determine the possible moves based on the board state. The idea behind ML is to have the program teach itself by iterating its internal logic using all the data available as input, gradually refining its results towards a predefined goal (RUSSEL, 1995).

The modern society produces an incredible amount of data each day, being it originated from smartphones and computers, applications and social media or even antennas and satellites. With an unending stream of data to be feed, ML systems are discovering new ways of selling and advertising products, helping business owners to target audiences, helping managers make decisions and even helping with the test of new medicines (DOMINGOS, 2015).

\subsection{Reinforcement Learning}

Reinforcement Learning (RL) is a subfield of Machine Learning that is focused on how to teach agents the best actions to take on a specific scenario, which is done by letting the agent take an action and then analyze the outcome, compared to what is considered a good result. The idea is that, with each cycle of action, reward and analysis, the agent will gradually understand which actions produce the best outcome, gradually steering toward those actions and making more efficient choices.

Reinforcement Learning was responsible for many great feats in the latest years, being considered a buzzword in the IT community. As expected, this influx of interest also attracted many new followers, researchers and investors to the area, looking forward to discoveries and new opportunities (SHAIKH, 2017).

\subsection{Artificial Intelligence in video games}

Artificial Intelligence is an area inside the game industry responsible for making the games look smart, like producing enemies that behave with human nature, making reasonable pathfinding decisions or simply choosing the best tool for a specific situation. Many high-profile games have some sort of internal AI responsible for particular tasks, but before 1990 all of them used similar techniques, which relied on hard-coded choices based on if-else decisions that determined the next action. During the '90s games did still relied on defined states but started to introduce improved techniques, such as sense simulation, which allowed enemies to notice things such as dead friends and react accordingly (MILLINGTON, 2009). 
During the next years, other advancements were made, such as strategy games introducing noticeable AI pathfinding for units and formation motion for groups, while in the 2000s some games presented things such as neural network-based units. Some other topics still were not solved then, like RPGs using tree-based dialog systems or sports games having trouble with dynamic calculation required for match simulation.

While the need for complex AI depends on each video game, with some requiring an advanced AI to be enjoyable, others don't see much improvement by implementing a complex system, relying on basic solutions. The common use for AI is described by (MILLINGTON, 2009): "The AI in most modern games address three basic needs: the ability to move characters, the ability to make decisions about where to move and the ability to think tactically or strategically."

One example of Reinforcement Learning being at the spotlight was seen during a professional gaming tournament called The International, where a team of players fought each other competing for prizes of a total of US\$24,787,916. During the tournament a presentation was made, putting an agent trained with RL to play Dota 2 against the best Dota 2 players in the world: the result left the crowd ecstatic as the agent won easily against all human opponents (SHAIKH, 2017).

That particular agent was created by the nonprofit research company OpenAI, which trained it for over four months against itself in countless games, where it learned on its own how to play the game. During those four months, the agent (also called a bot) went from a completely clueless player that didn't know how to navigate the map, to being able to beat the top world player handily. In Figure 2 we show the agent matchmaking rating (MMR) evolution during the four-month training period before the tournament, which is a normalized ranking. In this metric, $100 \%$ is the best player in the world. We can thus observe the steady improvement of the trained agent into the top ranks (OPENAI, 2017).

Figure 2: Agent evolution graph.

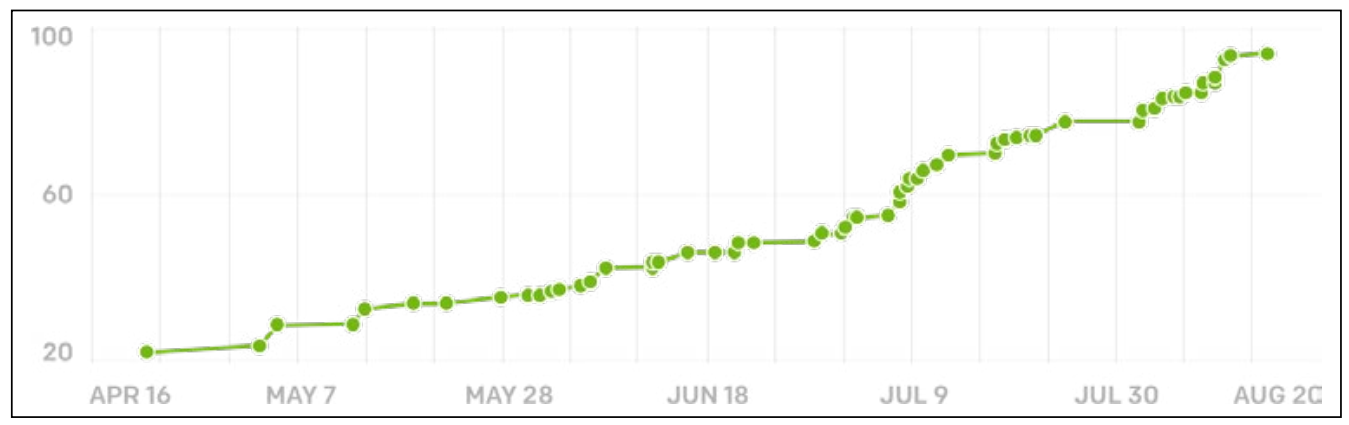

Source: (OPENAI, 2017) 


\section{DEVELOPMENT}

This section presents details relative to the tests, research, programming and results obtained during the experimentation phase. A special focus is given to neural network architectures, algorithms and their characteristics related to the discussed problems. Also, the tools that were used are presented, such as Starcraft II, SC2LE and TensorFlow.

\subsection{Game environment}

The first game of the Starcraft franchise was released in 1998 by Blizzard Entertainment, being highly successful. During its first years, it developed a strong community of developers, who built bots to fight each other in competitions.

Starcraft II (SC2) is the second game of the franchise, released in 2007. It was also considered a success. By being more complex, it offers the following challenges to RL agents:

- Two resources that need to be mined and managed (minerals and gas).

- Construction of production buildings.

- Different units and buildings, each with their actions and options.

- Completely different races.

- Large maps with different terrain levels.

- Land and air units.

- Training of an army.

- Management of hundreds of units during battle.

- Imperfect information due to the fog-of-war effect.

- Decisions that may only see their consequences later in the game.

\subsubsection{SC2LE}

Starcraft II Learning Environment (SC2LE) is a set of tools to help the community build their bots, resulting from the partnership of the developer with the Deepmind research company (VINYALS, 2017).

SC2LE is composed of the SC2client-proto, a Machine Learning API created by Blizzard that allows direct control of the game. It also contains the PySC2, which is a toolset written in Python that facilitates the use of the Blizzard API with the agents. There are also packs of replays from SC2, with more than 500 thousand replays to be used for Reinforcement Learning. Finally, there are mini-games aimed to help with the research of specific areas of the game, removing overall complexity when compared to the whole game. 
Figure 3 shows a representation of the SC2LE components during its use by an agent, demonstrating the input, decision making and reward analysis flow. Figure 4 shows an image of the game broken down into layers, identifying the various kinds of information necessary for the bots to make decisions.

Figure 3: Representation of SC2LE flow.

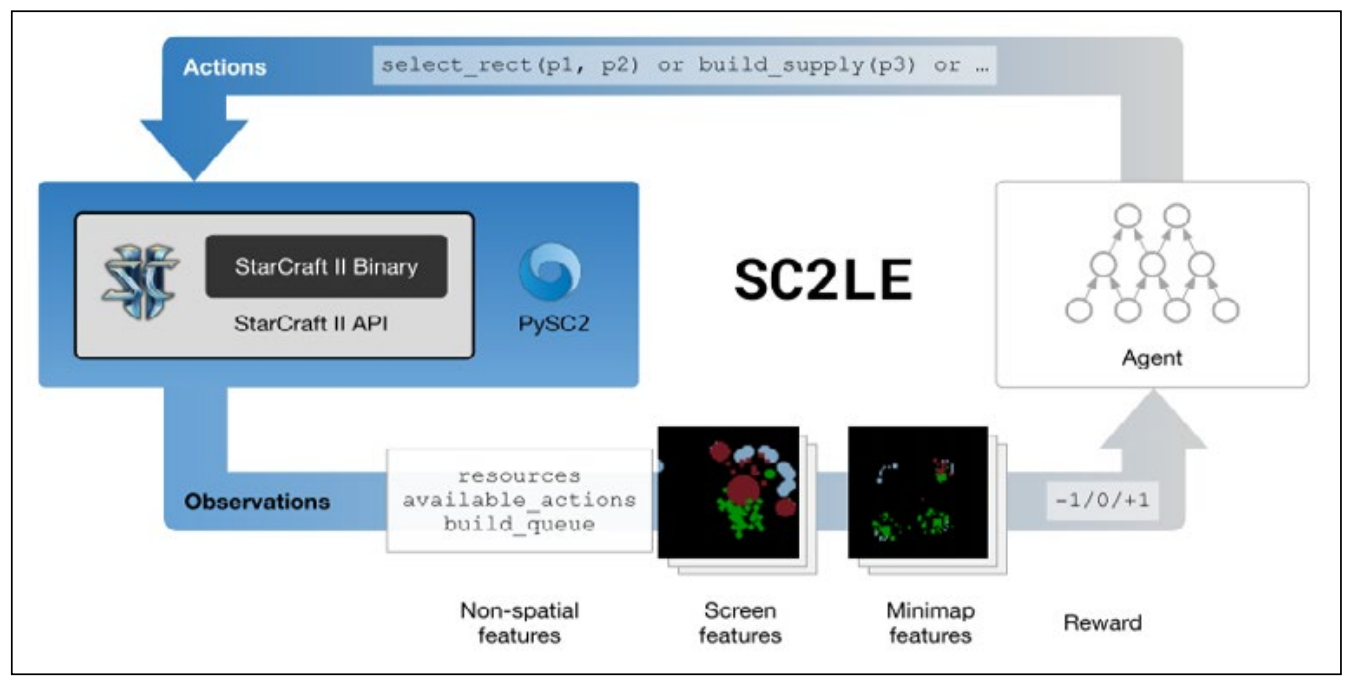

Source: (VINYALS, 2017)

Figure 4: Depiction of the different information layers.

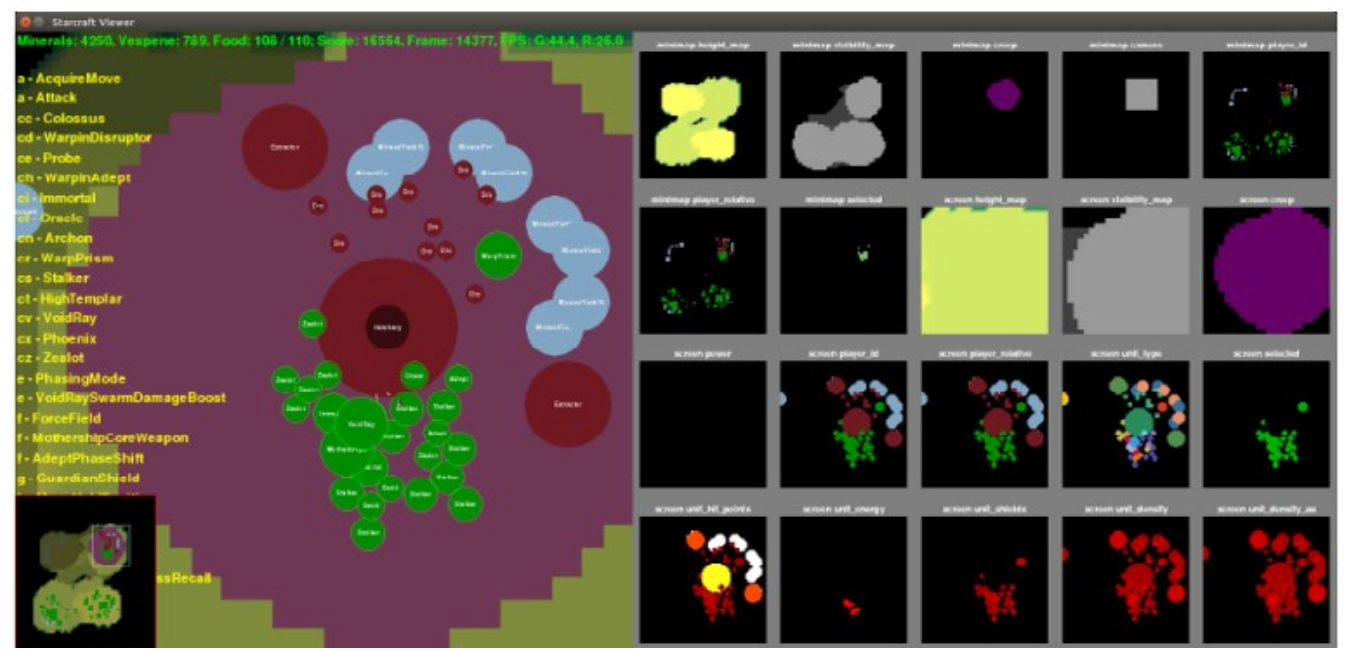

Source: (VINYALS, 2017) 


\subsubsection{Agents}

The SC2LE agents follow stages that are similar no matter which minigame or RL algorithm is being used. The process starts by reading the current game state and doing an analysis of the rewards received from the last execution (called an episode), which then is applied to the current neural network to choose the next action that is going to be taken.

The agent tested in this work was built using the Python language, using many packages available for its environment, such as the OpenAI Baselines. This structure allowed the agent to be created with a lean structure that only diverged when defining the underlying Reinforcement Learning algorithm. That is, most of the code was generic enough to provide a testbed for a comparison of distinct RL strategies.

\subsubsection{Mini-games}

SC2LE mini-games consist of small Starcraft II maps that isolate elements of the full game, allowing for agents to be created and tested in a much simpler environment. Those mini-games break the full game into smaller tasks such as moving into specific locations, collecting resources such as minerals and gas, building units, defeating enemies and finding the enemies themselves. An example of a mini-game session can be seen in Figure 5.

Figure 5: Mini-game being played.

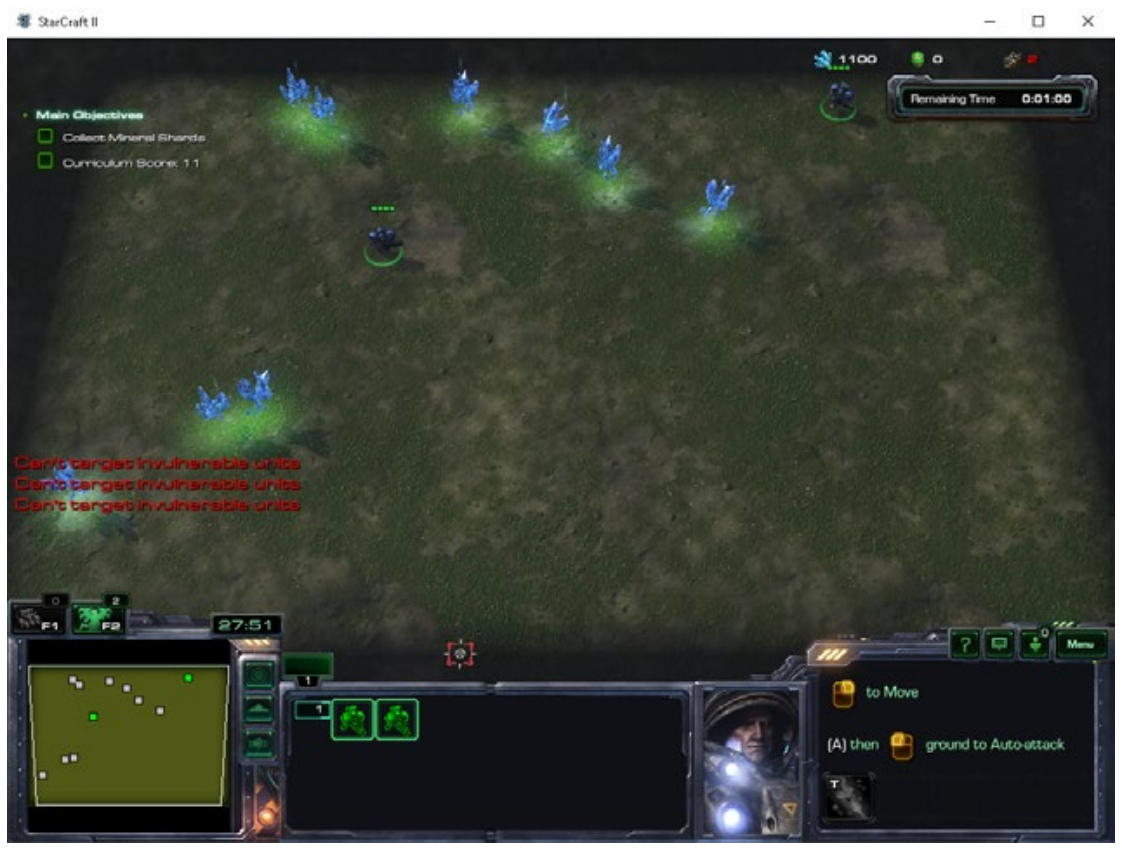

Source: from the authors (2019) 
One mini-game used was the Collect Minerals, which is one of the most basic tasks required to successfully play the game. It challenges the bot to collect game resources as efficiently as possible while controlling two individual units at the same time. In this map, the agent starts with two marine units and must use them to collect all 20 mineral shards.

Figure 6: Scores by episode.

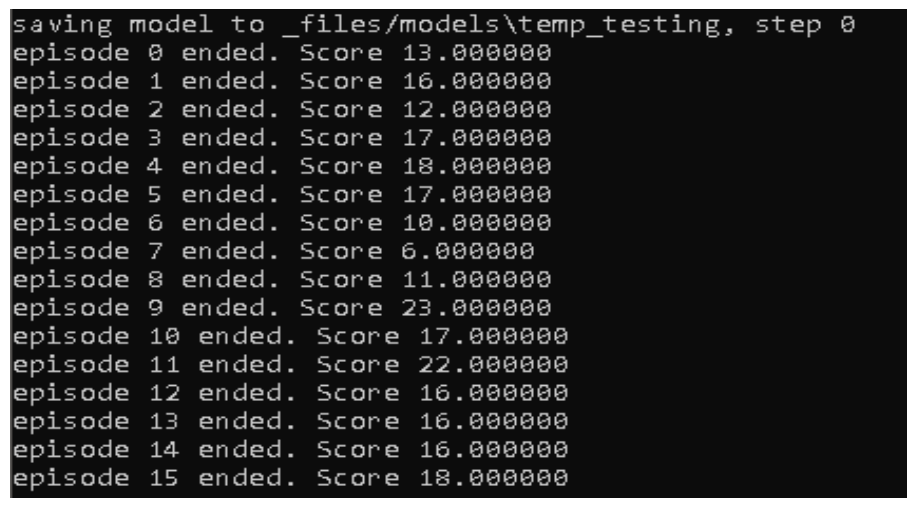

Source: from the authors (2019)

Each episode of the mini-game lasts for 120 seconds, a time during which the agent will try to collect as many minerals as possible. If all the minerals are collected, a new set of 20 is spawned. The final score of the agent is based on the minerals collected, which can be seen on the top left corner of the screen or printed to the console, as shown in Figure 6. 
Figure 7: Mini-game being played.

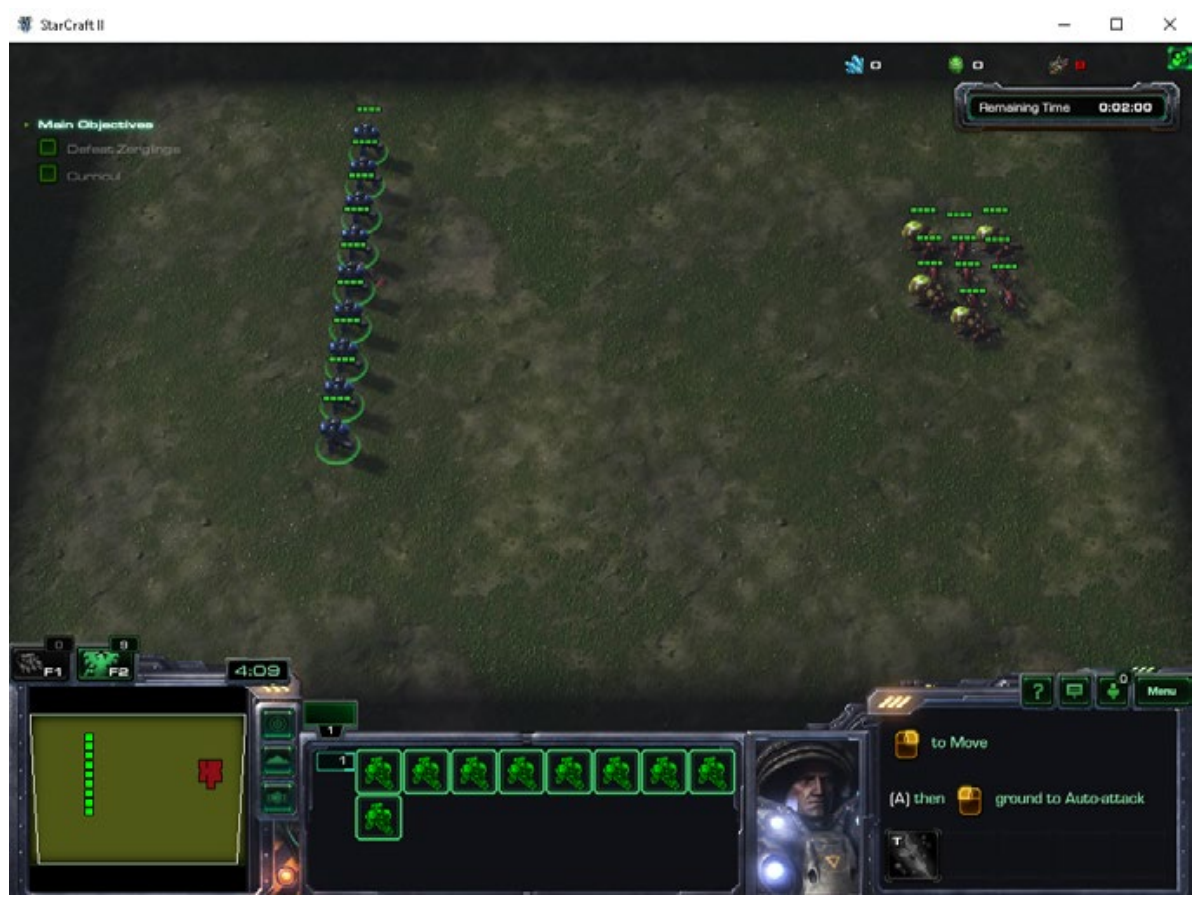

Source: from the authors (2019)

Another mini-game used was the Defeat Zerglings and Banelings, which mimics the combat mechanic from the game, requiring the agent to use the units available to defeat the enemies. In this map, the agent starts with nine marine units and must defeat six zerglings units and four banelings units, as is shown in Figure 7.

Each episode of the mini-game lasts for 120 seconds, the time during which the agent will try to kill as many enemies as possible. If all the enemies are defeated, a new set of six zerglings and four banelings are spawned, whereas and the agent receives four extra marine units at full health. The final score of the agent is based on the number of enemies killed during the episode, which can be seen on the top left corner of the mini-game screen or likewise printed to the console.

\subsection{Machine learning tools}

TensorFlow is a software library for numerical computation through graphs. Nodes represent mathematical operations and the edges represent the data arrays transferred between nodes. It was created by the Google Brain project, being a general enough architecture to be applied in a wide range of cases and domains. 
The OpenAI Baselines is a collection of high-quality Reinforcement Learning algorithms, already being vastly used in many academic studies and commercial projects. From the available implementations, the Advantage Actor-Critic (A2C) and the Deep Queue Network(DQN) were selected for being the most commonly used in academic projects.

\subsubsection{Advantage Actor Critic - A2C}

In general, the idea for Reinforcement Learning algorithms is to have an agent that receives the state of the environment and then takes actions while trying to maximize its rewards. The Advantage Actor Critic fulfills that premise in a few steps.

First, it receives the current state of the environment and uses it to generate two outputs. One output is the estimate of rewards he expects to find ahead, which is called 'state value' and is meant to be the 'critic'. The second output is a recommendation of what action to take, called 'policy', and which is meant to be the 'actor'.

Each step of state-action-reward results in the recording of the state, reward expected, action taken and reward found. After collecting the information for three iterations, the agent adjusts its critic based on the estimates he had before and the results collected. For that, it calculates the difference between what he expected, what he received and what lead him to take the action.

The algorithm also ensures that the agent won't always choose the safest option, which may have a low potential for rewards. That is done by subtracting a value called 'entropy' from the loss function, which is responsible for measuring the performance of the actions. A schematic implementation of the A2C model can be seen in Figure 8, using a robot as an example scenario.

Figure 8: Actor - Critic Model.

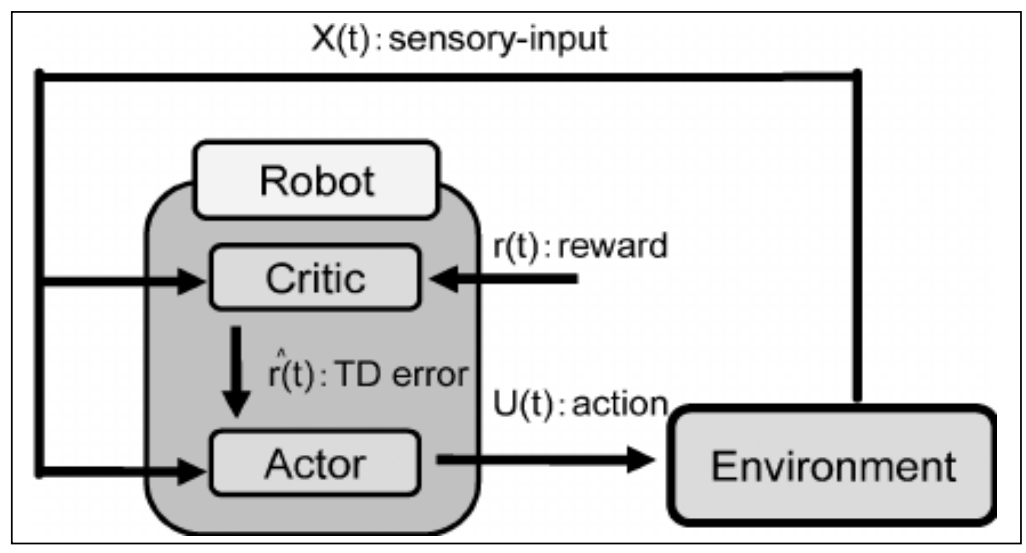

Source: (MAO, 2017) 


\subsubsection{Deep Queue Learning - DQN}

The Deep Queue Learning algorithm is a direct evolution from the Q-Learning algorithm. It was created to solve the lack of generality of the Q-Learning, which weakened its utility. So even though Q-Learning is a very good RL algorithm, such a lack of generality makes it unable to estimate the outcome values for states that it has not yet seen.

DQN improves the Q-Learning algorithm by introducing a neural network to estimate the reward values, giving it the ability to store the results already obtained by specific action-state decisions taken. Another improvement is the fact that the DQN implementation uses randomly picked batches of experiences from its pool, to help the network to develop itself with a broad range of experiences.

The third addition to the Q-Learning implementation is the use of a second network during the training process, responsible for reward estimates that are used in the loss function during the training process. By having separate networks for each area, DQN reduces the risk of estimations values spiraling out of control, resulting in a feedback loop of state, reward estimates and actions taken. A schematic implementation of the DQN model can be seen in Figure 9.

Figure 9: Deep Queue Network Model.

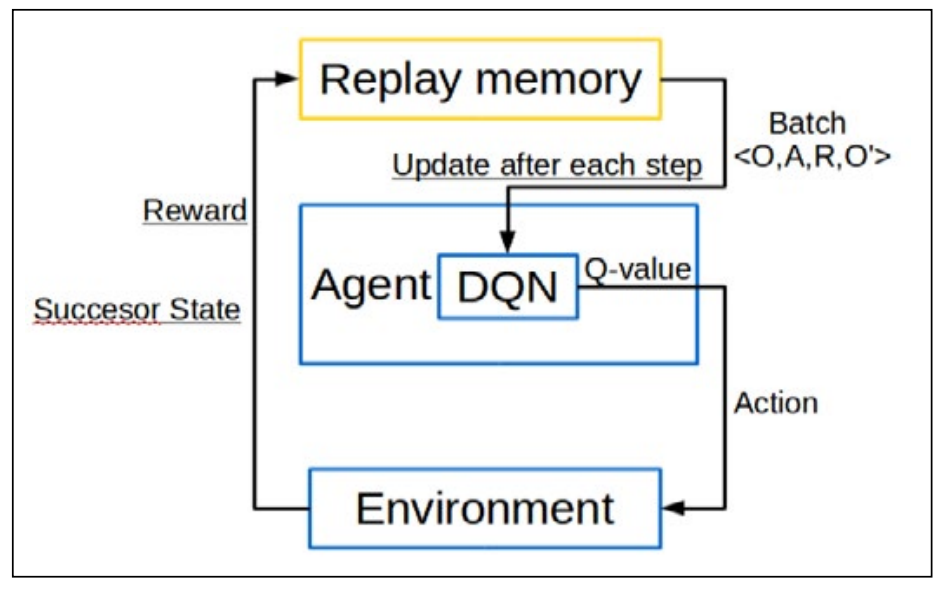

Source: (LEE, 2018)

\subsection{Communication}

The setup necessary for the training requires that multiple separate parts work together, such as the communication between the agent and the game, which allows the agent to send commands to the game and receive the current game state. 
The SC2client-proto is a Machine Learning API created by Blizzard, being an interface that offers full control of the game, exposing all the necessary commands and information for bots and replay analyzers to work.

To facilitate the task of creating agents that play SC2, DeepMind created the PySC2, which consists of a set of tools that exposes SC2client-proto API as a Python RL environment package. It provides an interface for developers to create the agents without the need to use direct commands to the game, offering an easy-to-use wrapper for the RL agents to interact with SC2.

The mini-games being used for the agents training consist of SC2 custom maps that were created with specific sets of rules, and can only be run using the SC2 game client. SC2 maps are created using the Blizzard Map creator tool and all the data related to each map is stored in a 'SC2Map' format file.

\section{TRAINING}

The training was divided into two groups, defined by the mini-game being played, with each group of tests being composed by sessions of the agent playing the mini-game with both RL algorithms and the learning parameters being tweaked.

Each training session was limited to 100 episodes of the mini-game being played by the agent, focusing the attention on the score value obtained by an algorithm and its specific parameters during the session. Each training session data generated was collected as well as the trained model, which allowed the analysis of the score by episode, as well as the use of the pre-trained agent model on side-by-side tests with other agents.

\subsection{Agent Setup}

The agent has a set of parameters that defines some aspects of its behavior during training, those parameters being independent of the RL algorithm being used. The relevant parameters are the following:

- Discount: reward discount for the agent. It can be tweaked to test the reward influence during training;

- Loss Value Weight: how much a loss weights. It can be tweaked to test the influence of a loss during the training;

- Entropy: correspond to the spread of action probabilities, where low entropy means one dominant action while high entropy means multiple actions with similar probability. It can be tweaked to test how it affects the agent's exploration of new actions or strategies.

The agent setup for the training sessions was defined as one set with default values for all described parameters for both algorithms and another set with an altered entropy value from $1 \mathrm{e}-6$ to $1 \mathrm{e}-5$ for both algorithms, as described in Table 1. 
Table 1: Agent Parameters Setup Table

\begin{tabular}{|l|c|c|}
\hline & Default Setup & Custom Setup \\
\hline Discount value & 1 & 1 \\
\hline Loss Weight value & 1 & 1 \\
\hline Entropy value & $1 \mathrm{e}-6$ & $1 \mathrm{e}-5$ \\
\hline
\end{tabular}

Source: from the authors (2019)

\subsection{Results}

This section presents the results collected, showing the most significant values.

\subsubsection{Defeat Zerglings mini-game}

The first test was conducted using the A2C algorithm with default parameters. The scores obtained by the agent ranged from 1 to 82 , resulting in the graph seen in Figure 10.

Figure 10: A2C results with default parameters for Defeat Zerglings: the vertical axis represents the score obtained, while the horizontal axes indicates the 100 individual episodes.

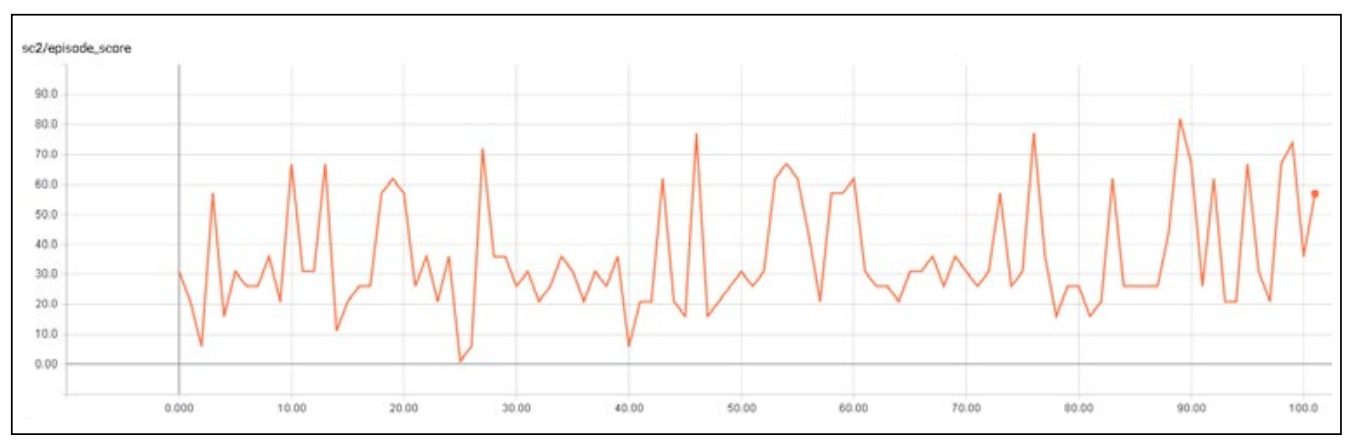

Source: from the authors (2019)

The second test was conducted using the DQN algorithm with default parameters. The scores obtained by the agent ranged from 1 to 77 , resulting in the graph seen in Figure 11. 
Figure 11: DQN results with default parameters for Defeat Zerglings: the vertical axis represents the score obtained, while the horizontal axes indicates the 100 individual episodes.

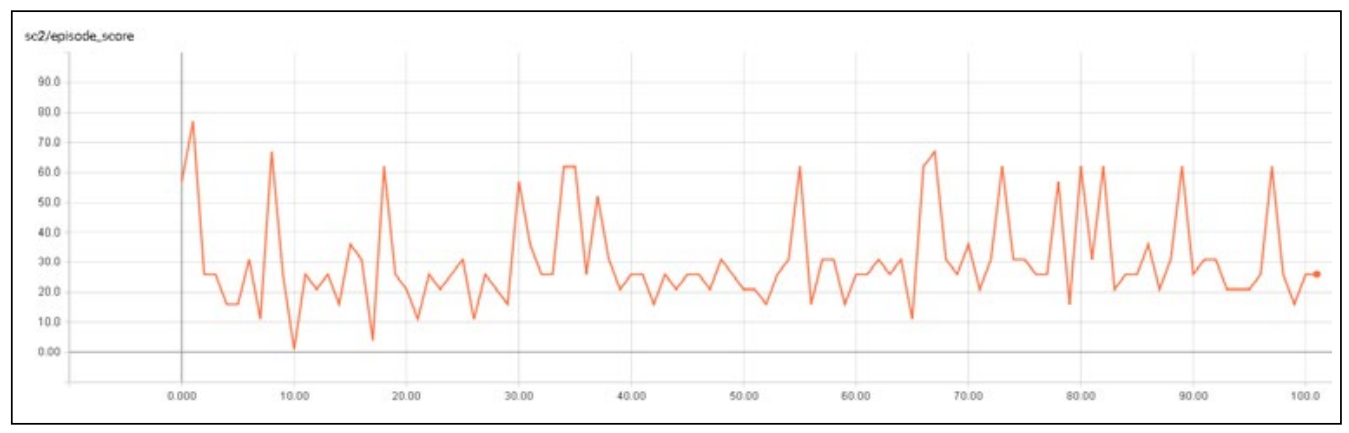

Source: from the authors (2019)

The third test was conducted using the A2C algorithm with custom parameters. The scores obtained by the agent ranged from 1 to 77 , resulting in the graph seen in Figure 12. The fourth test was conducted using the DQN algorithm with custom parameters. The scores obtained by the agent ranged from 6 to 93, resulting in the graph seen in Figure 13. In Table 2 the values obtained during the 'Defeat Zerglings' mini-game are presented.

Figure 12: A2C results with custom parameters for Defeat Zerglings: the vertical axis represents the score obtained, while the horizontal axes indicates the 100 individual episodes.

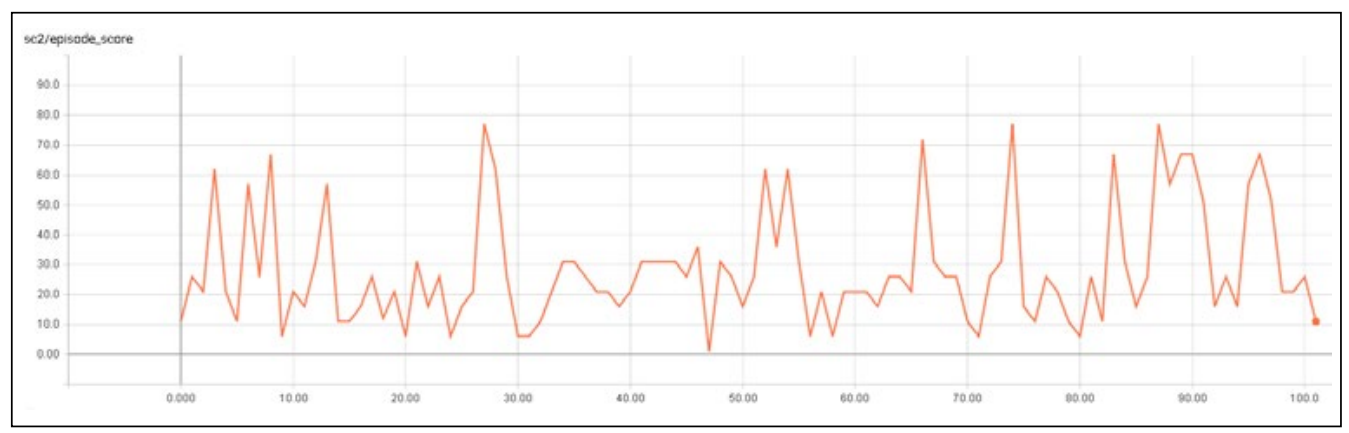

Source: from the authors (2019) 
Figure 13: DQN results with custom parameters for Defeat Zerglings: the vertical axis represents the score obtained, while the horizontal axes indicates the 100 individual episodes.

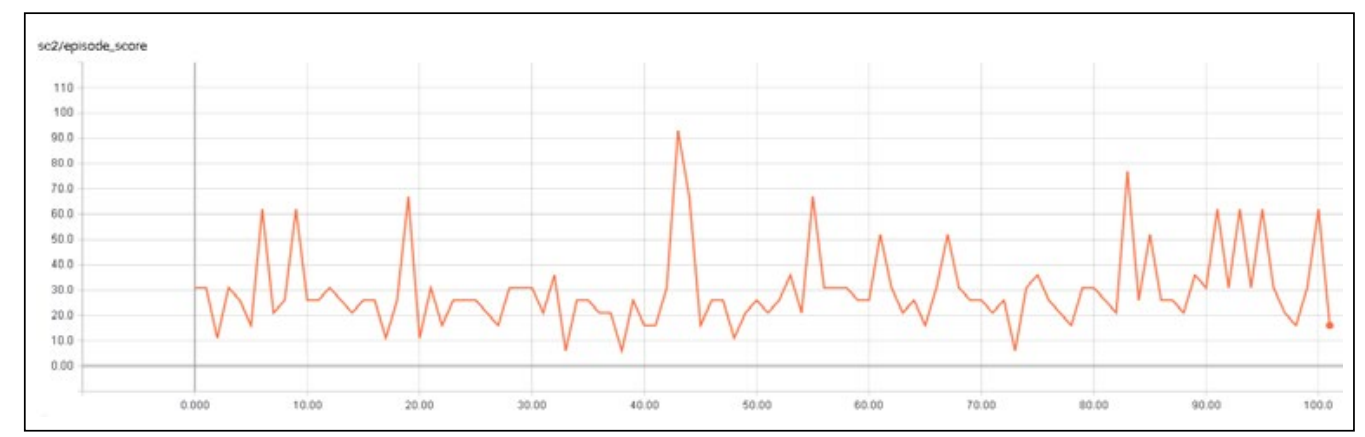

Source: from the authors (2019)

Table 2: Defeat Zerglings Results Table.

\begin{tabular}{|l|c|c|c|c|}
\hline & A2C default & DQN default & A2C custom & DQN custom \\
\hline lowest value & 1 & 1 & 1 & 6 \\
\hline highest value & 82 & 77 & 77 & 93 \\
\hline average value & 35,21 & 30,6 & 28,59 & 29,69 \\
\hline
\end{tabular}

Source: from the authors (2019)

\subsubsection{Collect Minerals mini-game}

The first test was conducted using the A2C algorithm with default parameters. The scores obtained by the agent ranged from 10 to 29 , resulting in the graph shown in Figure 14. 
Figure 14: A2C results with default parameters for Collect Minerals: the vertical axis represents the score obtained, while the horizontal axis indicates the 100 individual episodes.

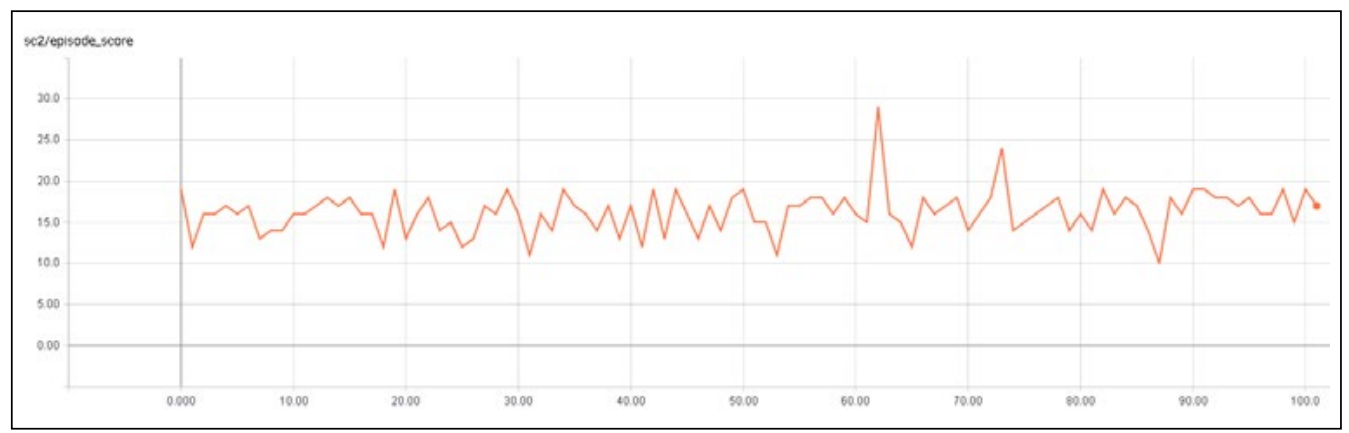

Source: from the authors (2019)

The second test was conducted using the DQN algorithm with default parameters. The scores obtained by the agent ranged from 10 to 24 , resulting in the graph seen in Figure 15.

Figure 15: DQN results with default parameters for Collect Minerals: the vertical axis represents the score obtained, while the horizontal axis indicates the 100 individual episodes.

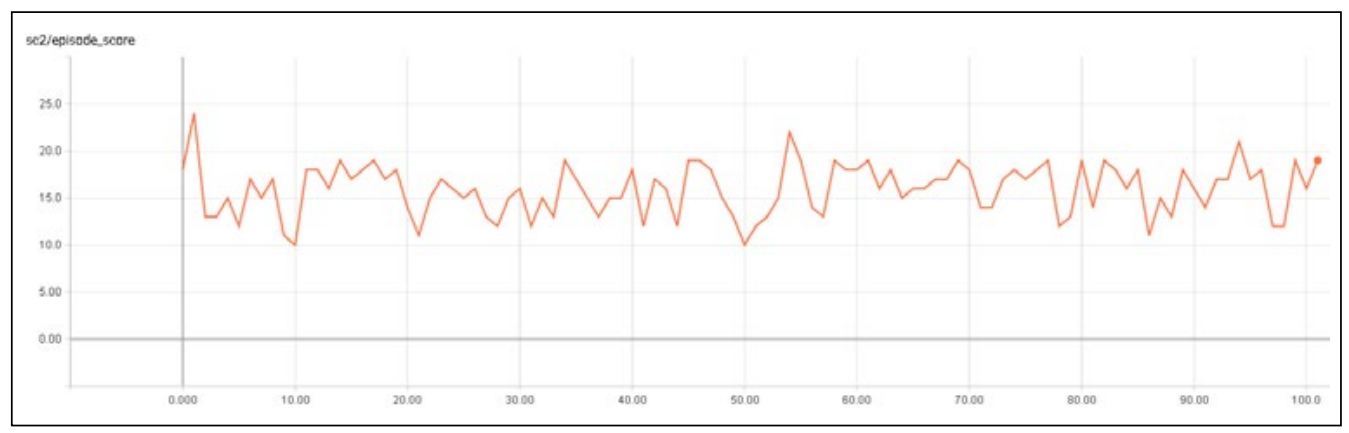

Source: from the authors (2019)

The third test conducted was the 'Collect Minerals' mini-game using the $\mathrm{A} 2 \mathrm{C}$ algorithm with custom parameters, where the scores obtained by the agent ranged from 8 to 36 . The fourth test conducted was the 'Collect Minerals' mini-game using the DQN algorithm with custom value parameters, where the scores obtained by the agent ranged from 7 to 33. These graphs are not shown for brevity.

In Table 3 are presented the lowest, highest and average values obtained by each algorithm during the 'Collect Mineral' mini-game. 
Table 3: Collect Mineral Shards Results Table.

\begin{tabular}{|l|c|c|c|c|}
\hline & A2C default & DQN default & A2C custom & DQN custom \\
\hline lowest score & 10 & 10 & 8 & 7 \\
\hline highest score & 29 & 24 & 36 & 33 \\
\hline average score & 16,18 & 15,88 & 17,07 & 16,65 \\
\hline
\end{tabular}

Source: from the authors (2019)

\subsection{Analysis}

The results from the "Defeat Zerglings' mini-game showed overall similar results for the $\mathrm{A} 2 \mathrm{C}$ and $\mathrm{DQN}$ algorithms, with the $\mathrm{A} 2 \mathrm{C}$ faring better with default value parameters while the DQN did better on the custom ones. The entropy parameter changes drove the DQN scores up and the A2C down, resulting in a bigger difference range between lowest and highest scores, which resulted in lower score average values.

The results from the Collect Minerals mini-game showed that the A2C algorithm fared better than the DQN on both the default and custom parameters tests. The entropy parameter changes seemed to have influenced the resulting values, resulting in a bigger difference range between the lowest and highest scores, which also resulted in higher score average values for the custom setup.

Considering the few results already obtained, the $\mathrm{A} 2 \mathrm{C}$ seems to offer the best results when used for the Reinforcement Learning agents in this particular setup of Starcraft II mini-games. The parameter change during the 'Defeat Zerglings' that resulted in the DQN doing better at that test indicates that even a slight adjust in the parameters can influence the final results, for best or worst.

The test consisted of a total of eight training sessions, four on each minigame, being two with each algorithm, one with default parameters and one with custom parameters. Each Collect Minerals training session took close to one hour, while the Defeat Zerglings varied between 30 to 50 minutes because the episodes could end sooner if the agent lost all its units.

The machine used for the tests was a low-end desktop configuration, with an AMD Phenom II 955 running on $3.200 \mathrm{MHz}$ clock and using $8 \mathrm{~Gb}$ of DDR3 RAM running on $1333 \mathrm{MHz}$. Taking into consideration the computer specs, it's reasonable to affirm that the time taken for the test could be considerably lower using a more computationally powerful machine.

\section{CONCLUSION}

This work has briefly analyzed the current state of two Reinforcement Learning algorithms applied to a complex and modern computer strategy game. Tests were conducted using default and custom agent parameters, 
offering some preliminary answers about how each algorithm would achieve success on each mini-game and how the parameters might affect them.

While each test session only amounted for a small number of episodes, we confirmed that changes in the parameters and the algorithm being used on each mini-game do significantly affect the results, even without the agent achieving a convergence point.

The entropy being selected to be the changed parameter showed us that it can have a heavy influence on the results, leaving open the question about how the other parameters would affect the tests.

Related works focus on convergence, comparing algorithms and the time required for each to achieve the convergence point, while this work focused more on fine comparison of algorithm results and parameters on a smaller range of episodes.

\subsection{Future Work}

This work used a reduced amount of episodes for each training session, which may explain the sharp changes in behavior based on the parameters and algorithm being tested.

A future evaluation may be done on dedicated servers with parallel hardware optimizations for mathematical calculations, which could extend the training sessions by a large amount, being able to achieve convergence in results as the agents learn the best strategies for each mini-map.

Another interesting task is the study of Reinforcement Learning algorithms that were not tested in this work, followed by the challenge of training agents to play the whole game, which would require it master all of the tasks previous seen individually in the mini-games.

\section{REFERENCES}

ADAMS, R. Ten powerful examples of Artificial Intelligence in use today. Available at: <https:/ / www.forbes.com/sites/robertadams/2017/01/10/10-powerfulexamples-of-artificial-intelligence-in-use-today/>. Accessed on October 232017.

ATTICK, R. Intelligent Things: It's all about machine learning. Available at: $<$ https:/ / www.linkedin.com/pulse/intelligent-things-its-all-machine-learning-roger-attick//>. Accessed on October 22, 2017.

DOMINGOS, P. The Master Algorithm: How the Quest for the Ultimate Learning Machine Will Remake Our World. Basic Books, 2015.

LEE, W. Deep Q Networks. Available at: <https:/ /dnddnjs.gitbooks.io/rl/content/ deep_q_networks.html>. Accessed on June 14, 2018. 
MAO, H. Reinforcement Learning using Asynchronous Advantage Actor Critic. 2017. Available at: <https:/ / medium.com/@henrymao/reinforcement-learningusing-asynchronous-advantage-actor-critic-704147f91686>. Accessed on June 14, 2018.

MILLINGTON, I. Artificial Intelligence for Games. Morgan Kaufmann Publishers, 2009.

NARULA, G. Everyday Examples of Artificial Intelligence and Machine Learning. Available at: <https:/ / www.techemergence.com/everyday-examples-of-ai/>. Accessed on October 22, 2017.

NIELSEN, M. Using neural nets to recognize handwritten digits. Available at: $<$ http:/ / neuralnetworksanddeeplearning.com/chap1.html >. Accessed on October 25, 2017.

OPENAI. GYM: A toolkit for developing and comparing reinforcement learning algorithms. Available at: <https://github.com/openai/gym/>. Accessed on October $25,2017$.

RUSSEL, S.; NORVIG, P. Artificial Intelligence - a modern approach. Prentice-Hall, New Jersey, 1995.

SHAIKH, F. Simple Beginner's guide to Reinforcement Learning \& its implementation. Available at: <https:/ / www.analyticsvidhya.com/blog/2017/01/ introduction-to-reinforcement-learning-implementation/>. Accessed on October 25, 2017.

VINYALS, O., et al. Starcraft II: A new challenge for reinforcement learning. arXiv preprint arXiv:1708.04782 (2017). 